\title{
Incorporación de estándares bioéticos para la generación de conocimiento científico de calidad en investigación en fauna silvestre: Ciencia con conciencia
}

\author{
Incorporation of bioethical standards for the generation of high quality scientific knowledge \\ from research in wildlife: Science with conscience
}

\section{Tamara A. Tadich ${ }^{1,2}$, Inés de Freslón ${ }^{3}$, Carmen Gallo ${ }^{3,4}$, Jesús M. Zúñiga ${ }^{5}$, Ronald Vargas ${ }^{2}$, Cristian G. Torres ${ }^{1}$, Néstor Tadich ${ }^{3}$, Jessica Gimpel ${ }^{6,15}$, Claudio Martinez ${ }^{7}$, Daniel Sandoval, Ricardo Enríquez ${ }^{9}$, Julio Alfaro ${ }^{10}$, Pablo Muñoz ${ }^{11}$, Rodolfo Paredes ${ }^{12}$, Benjamín Erranz ${ }^{13}$, Ingrid Carvacho ${ }^{14}$, Marcelo Mezzano ${ }^{15}$, Emilio A. Herrera ${ }^{2,16^{*}}$}

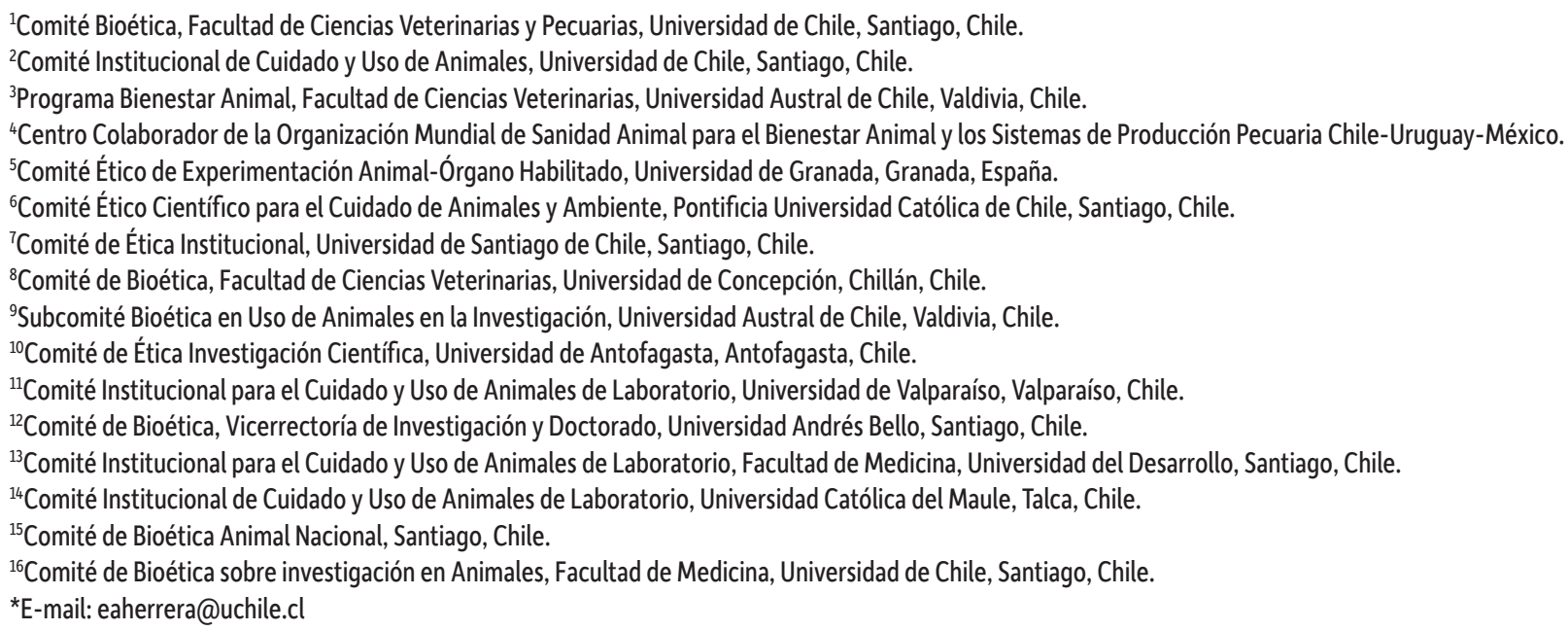
ambiental y generación de conocimiento. El objetivo de los Comités Institucionales de Cuidado y Uso de Animales (CICUAs) es que dicha investigación se realice con altos estándares de bioética y bienestar animal, minimizando el sufrimiento animal y el impacto ambiental. Este artículo destaca la importancia de los CICUAs como un aliado para adecuar los procedimientos con animales a la normativa y facilitar el avance científico.

Palabras clave: bienestar animal, bioética, CICUAs, experimentación animal.

\section{ABSTRACT}

Research using wild animals has diverse aims, such as conservation, environmental management, and the generation of knowledge. The aim of the Institutional Animal Care and Use Committees (IACUCs) is to assure that such research is carried out under high bioethical and animal welfare standards, minimizing animal suffering and environmental impact. This manuscript highlights the relevance of IACUCs as an ally to adjust the procedures on animals to the norm and thus facilitate scientific progress.

Keywords: animal experimentation, animal welfare, bioethics, IACUCs. 


\section{INTRODUCCIÓN}

Existen diversas actividades humanas que afectan a los animales. Tales acciones deben realizarse con responsabilidad y respeto, teniendo como prioridad el bienestar animal. De acuerdo a Fraser y MacRae (2011), estas actividades pueden clasificarse en cuatro tipos:

1) mantención y manipulación de animales con fines de compañía, producción, investigación o exhibición;

2) actividades humanas que causan daño deliberado a los animales como el control de plagas, faena, caza y ensayos toxicológicos;

3) actividades que causan daño directo, pero no intencional, a los animales como el transporte, sistemas agrícolas, contaminación lumínica; y

4) actividades que causan daño indirecto a través de la perturbación de los sistemas ecológicos y de procesos naturales, como la destrucción de hábitats, introducción de especies invasoras y contaminación.

Solo en el caso de 1) y 2), los Comités Institucionales de Cuidado y Uso de Animales (CICUAs), o Comités de Bioética Animal, tienen injerencia (fines científicos), ya sea mejorando las condiciones de mantención o disminuyendo el sufrimiento innecesario asociado a la manipulación. Para las actividades asociadas a los puntos 3) y 4), existen otros mecanismos de control, ejercidos por instituciones, leyes y autoridades. Es en estos últimos dos puntos donde se considerarían las otras actividades humanas que pueden afectar el estatus de las poblaciones naturales, como aquellas de origen antrópico (e.g. actividad forestal) descritas por D'Elia et al. (2019).

Toda actividad asociada al manejo de fauna silvestre puede generar dilemas éticos. En este manuscrito nos centraremos en las cuestiones relativas a cómo el bienestar animal puede verse afectado por prácticas de manejo realizadas con fines de investigación científica.

Los estudios de campo con animales silvestres conllevan potenciales riesgos para el bienestar de los sujetos de estudio. Este impacto puede ser tan alto o mayor que el causado por los estudios realizados con modelos animales estandarizados en condiciones de laboratorio, problemas que han sido poco considerados por parte de algunos colectivos de investigadores del área, básicamente por dos motivos:

(1) Los animales salvajes experimentan, de modo natural, niveles considerables de sufrimientos causados por el entorno (e.g. hambre, heridas, enfermedades, depredación).

(2) Gran parte de la investigación que se realiza con fauna silvestre está dirigida a la conservación, es decir orientada al bien de las poblaciones o especies, y por tanto el bienestar individual es considerado como de importancia secundaria.

En resumen, la investigación con Fauna Silvestre (FS), presenta una problemática única tanto desde el punto de vista bioético como normativo y logístico, que debe ser analizada con objetividad y rigor (Reed et al. 2017; https://www.nc3rs. org.uk/wildlife-research).

¿Qué son y para Qué existen los Comités Institucionales de Cuidado y Uso de Animales (CICUAs)?

El uso de animales con fines de investigación impone deberes éticos, científicos y legales, para las instituciones, las entidades financiadoras que los apoyan, los/as investigadores/as responsables de ejecutarla, y la entidad que difunde los resultados. Por lo tanto, se debe asegurar a través de Programas de Cuidado y Uso de Animales, que el diseño de dichos estudios esté basado en nuestro mejor entendimiento de las necesidades biológicas y conductuales de los animales involucrados. De esta forma, se busca garantizar un trato humanitario que minimice posibles sufrimientos, en armonía con la obtención de resultados válidos y reproducibles (NRC, 2011).

Bajo esta necesidad es que surgen los Comités Institucionales de Cuidado y Uso de Animales, conocidos como CICUAs, o Comités de Bioética Animal (CBA). Estos Comités se denominan Institutional Animal Care and Use Committees (IACUC) en EE.UU. y como Comités Éticos de Experimentación Animal (CEEA) para la Unión Europea (UE), los que están legalmente establecidos (NRC, 2011; Directiva 2010/63/UE, 2010), y tienen por objetivo supervisar y evaluar continuamente el Programa de Cuidado y Uso de Animales. Además, deben revisar y aprobar las propuestas sobre el uso de animales, inspeccionar periódicamente las instalaciones y áreas donde se trabaja con animales, y responder a las inquietudes que pueda tener la comunidad sobre temas asociados al uso de animales con fines científicos, entre otras funciones (NRC, 2011). El fin último de estos comités es facilitar el avance de la ciencia y la generación de conocimiento, velando por el bienestar animal en los modelos experimentales y como consecuencia por la calidad y validez de los resultados obtenidos. En países con un mayor avance científico, estos Comités tienen la categoría de Organismos Habilitados por la autoridad competente, siendo veladores del cumplimiento de la legislación vigente, tanto en la comunidad científica como en las instituciones patrocinantes. 
En la Guía de Cuidado y Uso de Animales de la NRC, se sugiere que la constitución de los comités represente la diversidad de visiones de la comunidad académica (con y sin experiencia en investigación en animales) y civil. Esto favorece a que las sugerencias que pueda realizar un CICUA sobre la información declarada por los investigadores en sus protocolos, se basen en criterios científicos y principios éticos. Dichos principios están reconocidos en recomendaciones internacionales y nacionales, y en la legislación vigente de cada país (National Research Council-NRC, Organización Mundial de Sanidad Animal-OIE, National Centre for replacement refinement and reduction of animals in research-NC3Rs, Canadian Council on Animal Care-CCAC, Animal Welfare Act, Directiva 2010/63/UE y American Veterinary Medical Association-AVMA). Uno de estos principios globalmente aceptado y base para la mayoría de las normativas vigentes es el de las 3Rs. Estas normativas orientan la misión prioritaria de los CICUAs, la cual es asegurar el reemplazo animal siempre y cuando sea posible. En caso de que se justifique el uso de animales, los CICUAs aseguran minimizar el sufrimiento animal, independiente de la especie, a través de la reducción y el refinamiento.

¿Cú́l es la normativa que Regula las deCisiones de los CICUAs? A nivel internacional, existe legislación específica respecto al manejo de animales de experimentación, como es el Animal Welfare Act de 1966 en Estados Unidos (AWA 1966) o la Directiva 2010/63/UE relativa a la protección de los animales utilizados para experimentación y otros fines científicos. Estas y otras normativas, han sido utilizadas como base técnica para el apoyo de la creación y definición de funciones de los CICUAs en Chile.

En el año 2009, en Chile se aprobó la Ley Sobre Protección de Animales (Ley 20.380), donde se define "experimento en animales vivos" y se establecen reglas para su realización, junto con la disposición que crea un Comité de Bioética Animal permanente (CBA Nacional). A este Comité, conformado el año 2018, le corresponde definir las directrices bajo las cuales podrán desarrollarse los experimentos en animales vivos. Además de la normativa legal, existen directrices técnicas ampliamente aceptadas como la Guía para el Cuidado y Uso de Animales de Laboratorio (NRC, 2011). Otros documentos técnicos a considerar en Chile son el de "Regulación del uso y cuidado de animales en investigación" de la Comisión Nacional de Investigación Científica y Tecnológica (CONICYT) del año 2015 , organismo que a través de sus programas financia un importante porcentaje de la investigación que se realiza en el país y que involucra el uso de animales. En sus bases, CONICYT solicita una evaluación bioética de las propuestas a financiar a través de los fondos públicos que administra, respondiendo así a una creciente preocupación de la sociedad. Entendiendo el problema y la confusión que se genera con una exigua legislación nacional al respecto, el Fondo Nacional de Desarrollo Científico y Tecnológico (FONDECYT) ha tomado un rol preponderante y está permanentemente actualizando las recomendaciones y directrices técnicas y éticas a través de su Comité Asesor de Bioética.

Además de la Ley Sobre Protección de los Animales (20.380), existen otras disposiciones legales nacionales que regulan el manejo de animales, como la Ley CITES (20.962), Ley General de Pesca y Acuicultura (18.892), Ley de Caza (19.473) y sus reglamentos (Chile, 1989, 1996, 2009, 2016). Estas disposiciones se deben tener en consideración al momento de revisar y autorizar la investigación con animales.

ALGUNAS DIFERENCIAS ENTRE LOS MODELOS ANIMALES EN BIOMEDICINA Y LA INVESTIGACIÓN EN FAUNA SILVESTRE

Los animales de laboratorio (AL) están habituados, en cierta medida, a las circunstancias que ocurren en el laboratorio, aunque conservan su impronta filogenética. Por ejemplo, en su mayoría los AL presentan una historia de selección para ciertos rasgos morfológicos, fisiológicos y conductuales. Algunas líneas han sido seleccionadas para obtener modelos reproducibles de determinadas disfunciones $\mathrm{y} / \mathrm{o}$ patologías humanas (diabetes, cáncer, entre otras). Muchos de estos animales se consideran animales domésticos y existen pocas probabilidades de que pudiesen sobrevivir en condiciones naturales. Por su parte, los animales silvestres conservan sus rasgos de conducta, fisiología y morfología naturales (Lindsjö et al. 2016). Por esto, algunos manejos que parecieran inofensivos como, la captura, marcaje con pintura, anillamiento, colocación de equipos de monitoreo o tomas de muestra (sangre $\mathrm{u}$ otros tejidos) pueden generar sufrimiento animal, o cambios conductuales y problemas en los mecanismos de comunicación, aumentando por ejemplo la depredación sobre ellos (Swart, 2004). Por lo anterior, el manejo (y su refinamiento) de especies silvestres en condiciones naturales es más crítico que el de los modelos convencionales de animales de experimentación (Swart, 2004; Swart \& Keulartz, 2011).

En cuanto a los problemas de pérdida de bienestar, a ambos grupos (AL y FS) les afectan los mismos: (i) imposibilidad de llevar a cabo su repertorio conductual especie específico, por causas de la manipulación, inmovilización, restricción temporal o permanente de la conducta; (ii) generación de estrés y/o distrés por estímulos aversivos, (iii) y dolor generado por procedimientos experimentales. A estos efectos, en el caso de FS hay que añadir (iv) las interacciones animal/hombre, (v) el nivel de responsabilidad entre la población animal versus individuos objeto de estudio, (vi) la naturaleza del animal 
como modelo de respuestas biológicas, (vii) la reducción de su valor reproductivo (supervivencia, longevidad, fertilidad), y (viii) la generación de interferencias colaterales a especies diferentes de forma directa o indirecta (Swart \& Keulartz, 2011). Por ejemplo, se ha establecido que una mínima reducción de la competencia inmune, alimenticia, social o reproductiva en FS puede generar consecuencias severas a mediano y largo plazo (Redondo, 2012). En la práctica la mayoría de los procedimientos habituales que se realizan con animales salvajes resultan potencialmente dañinos desde el punto de vista del bienestar (Redondo, 2012).

Por otra parte, si bien puede existir investigación oportunista de especies silvestres, el bienestar del animal debe siempre ser considerado como primordial (Jessup, 2003). Es así como el Animal Research Review Panel (ARRP) ha desarrollado una guía a considerar tanto por los investigadores como por los CICUAs en estos casos (ARRP, 2019). Esta guía aplica a situaciones inesperadas que ocurren durante el trabajo en terreno, donde se presenta la oportunidad de tomar muestras de una especie no considerada en el protocolo aprobado por el CICUA. En este caso los investigadores deben informar de la modificación lo antes posible a su Comité, el cual deberá analizar el mérito de cada situación en particular. Procedimientos que no son considerados aceptables dentro de la investigación oportunista son la identificación por tatuajes y/o amputaciones de falanges, y en particular las de carácter invasivo como cirugías, transporte, restricción de movimiento por periodos prolongados de tiempo, colocación de sistemas de radio transmisión externos o implantados, y toma de muestras (Sikes et al. 2016; Lindsjö et al. 2016; ARRP, 2019).

\section{PRINCIPIO dE LAS 3R}

El principio de las $3 R$ involucra el reemplazo, reducción y refinamiento en el uso de animales de experimentación (Russell \& Burch, 1959), los que se describen a continuación:

Reemplazo. El reemplazo puede ser absoluto, buscando la sustitución de métodos que involucren experimentación con seres vivos y conscientes por material no sintiente (estudios in silico, in vitro), o parcial a través de la incorporación de especies con menor capacidad de sintiencia. A diferencia de la investigación biomédica los estudios en FS buscan obtener información sobre la biología, el comportamiento o la ecología de estas especies, por lo que el reemplazo no es posible.

Reducción. Abarca estrategias destinadas a obtener niveles comparables de información utilizando un menor número de animales o maximizar la información obtenida a partir de un número dado de animales. La reducción requiere de un diseño experimental apropiado, aplicación de tecnologías actualizadas, uso de métodos estadísticos adecuados, control de la variabilidad y por sobre todo de hipótesis formuladas de manera rigurosa. Esto busca finalmente evitar que el número de animales utilizados sea insuficiente para obtener resultados representativos y concluyentes, o bien innecesariamente alto. Los daños colaterales sobre individuos o especies distintas a la propuesta en el estudio también deben ser evaluados por un CICUA y por la autoridad competente en materia de protección de la vida salvaje, en el caso de Chile, el Servicio Agrícola y Ganadero (SAG).

Refinamiento. Se refiere a perfeccionar toda manipulación de animales, tales como mantención, reproducción y procedimientos experimentales, con el fin de procurar el bienestar animal y minimizar o eliminar el dolor y angustia (Russell \& Burch, 1959). El investigador, en conjunto con el CICUA, deben considerar cómo los procedimientos a realizar pueden afectar las condiciones fisiológicas, sociales, nutricionales, reproductivas y de vulnerabilidad de los animales; para así establecer métodos destinados a eliminar o reducir al mínimo este impacto negativo. Además, el investigador debe prever las posibles contingencias y determinar las medidas que se adoptarán para paliar los potenciales efectos negativos. Algunos aspectos a tener en cuenta son los requerimientos de los animales durante la captura, el transporte, alojamiento en cautividad, liberación, y las condiciones para interrumpir el procedimiento (criterio de punto final). Generalmente, la liberación sólo es recomendable cuando el procedimiento ha sido de severidad leve, de corta duración y el animal no ha perdido facultades físicas o de conducta (e.g. territorio, rango social, agilidad para escapar a predadores). En ocasiones, por ejemplo, si existe riesgo de transmisión de enfermedades a la población nativa, la liberación no resulta aconsejable (Crozier \& SchulteHostedde, 2014).

Tanto los investigadores como las instituciones que realizan investigación con animales, a través de sus CICUAs, deben tomar todas las medidas razonables para evitar el sufrimiento innecesario, basándose en la información científica disponible. Asimismo, en los casos que incluyan extracción definitiva del medio ambiente y eutanasia de animales, los métodos para tal procedimiento deben ser revisados por el CICUA para cautelar que correspondan a técnicas aceptadas y así evitar sufrimiento innecesario.

Finalmente, se debe considerar que los aspectos bioéticos aceptables son dinámicos y tanto investigadores como CICUAs deben actualizarse permanentemente. Por ejemplo, si en los años 70 se consideraba aceptable la identificación individual de anfibios con marca con fuego o la amputación de 
falanges (Honegger, 1979), hoy en día estos procedimientos no son aceptables, ya que existen alternativas que no generan sufrimiento ni cambios conductuales permanentes (refinamiento).

En particular, D'Elia et al. (2019) cuestionan el rol que debiesen tener los CICUAs en relación a los tamaños de muestra (reducción) y manipulaciones (refinamiento). En cuanto al cálculo del tamaño de muestra, en estudios en vida silvestre, en general los tamaños estimados son mayores que en modelos de AL. En parte, se puede explicar por la uniformidad somática, genética y fenotípica de los modelos animales estandarizados, frente a una potencial heterogeneidad de las variables analizadas en poblaciones naturales (Sikes et al. 2016). Otro factor que puede condicionar mayores tamaños de muestra es la condición de "Ceteris paribus" en animales silvestres, por la dificultad de mantener constantes todas las variables de una situación, menos las que se quieren estudiar (Swart, 2004).

Por lo tanto, es importante utilizar el mínimo número de animales estimado a través de métodos estadísticos confiables y que permita dar respuesta a la hipótesis, sin perder el rigor científico (NRC, 2011). Además, los investigadores que estudian FS son llamados a tomar las precauciones para minimizar eventos que generan miedo, distrés, o daño a largo plazo sobre los individuos estudiados, así como a minimizar el impacto sobre el ecosistema (e.g. trampeo de otras especies o by-catching) (Williams et al. 2002; Guidelines for the use of animals, 2012).

En el caso de la UE y otros países con legislaciones avanzadas, no sólo se aplica el principio de las $3 R$ s sino que además se pone a punto protocolos complementarios para maximizar el refinamiento de los procedimientos en base a los objetivos científicos. Por ejemplo, se está generalizando en las evaluaciones éticas la determinación obligatoria de la severidad de los procedimientos en base al grado de dolor, sufrimiento, angustia o daño duradero que se prevea que experimente un animal individual (Comisión Europea, 2012). Dichas determinaciones de severidad son totalmente aplicables en la investigación con FS y se han generado guías específicas y ejemplificadas que sirven de orientación a los investigadores (Porter, 1992; Barron et al. 2013; Carro et al. 2007).

Es así que la propuesta de D'Elia et al. (2019) de que "la manipulación de individuos terrestres debiese estar regulada principalmente por estándares de bienestar animal, pero no en relación al tipo de manipulación, número de animales y frecuencia temporal y espacial", y "Que la injerencia de los Comités de Bioética se limite a temas de bienestar animal relacionadas al refinamiento (e.g., manipulación en terreno, condiciones de mantención de animales en bioterios, modalidades de eutanasia) y bioseguridad; es decir que los Comités de Bioética no se involucren en relación al diseño de las investigaciones (e.g., tamaños muestrales, cobertura geográfica, tipo de muestra a tomar)." resultan contradictorias a los principios desarrollados por Russell y Burch (1959). Considerando que las 3 Rs contemplan el reemplazo (sustitución total o parcial), reducción (número de animales) y refinamiento (manipulación), y que estos tres principios deben ser abordados y justificados dentro del protocolo de cuidado y uso de animales, resulta impensable e inconsistente con las recomendaciones internacionales, que estos no sean revisados por los CICUAs. Probablemente dichas contradicciones en el uso de los términos técnicos son el resultado de una falta de comprensión en su totalidad de los criterios que fundamentan a las $3 R$ y su aplicación. Un diálogo fluido y transparente entre CICUAs e investigadores podría prevenir este tipo de contradicciones y malentendidos.

\section{GENERACIÓN DE CONOCIMIENTO CIENTífICO DE CALIDAD}

Actualmente nos encontramos frente a un escenario de "crisis de la reproducibilidad en la ciencia" (Loken \& Gelman, 2017), en gran parte dada porque históricamente no se ha exigido una claridad absoluta en la descripción de la metodología, de las características de los animales, ni de los métodos estadísticos aplicados (Kilkenny et al. 2010). Es por esto, que las instituciones que financian investigaciones, como el $\mathrm{NIH}$, han instruido a los comités científicos evaluadores de proyectos que aseguren rigurosidad en la revisión de los diseños experimentales (Begley et al. 2015). En Chile, FONDECYT también exige certificados de aprobación bioética al financiar proyectos que involucren el uso de animales. Por su parte, revistas como Science y Nature han actualizado sus guías incorporando checklists que permiten asegurar que los científicos estén utilizando los métodos apropiados, por ejemplo, para el cálculo de los tamaños muestrales mínimos (principio de reducción). También, las sociedades científicas como la Society for Conservation Biology, han publicado sus códigos de ética donde llaman a utilizar los estándares más altos en cuanto al trato de los animales, y a la utilización del método científico de manera rigurosa (Society for Conservation Biology, 2019). Actualmente se encuentran disponibles guías para reportar de manera estandarizada estudios con animales (ARRIVE), así como guías para planificar estudios con animales (PREPARE), las que incluyen recomendaciones para investigaciones de campo. La guía ARRIVE, originalmente publicada en Plos Biology (Kilkenny et al. 2010) ha sido respaldada por instituciones de investigación, fuentes de financiamiento y más de 1000 revistas científicas. Esta guía busca mejorar los estándares de la forma en que se comunican los resultados de estudios que involucran animales y permitir su reproducibilidad. Dentro 
de sus adherentes, destacan reconocidas revistas del área de la conservación, biología y ecología como Nature, Plos One, Plos Biology, BMC Ecology, Biological Research, Behavioural Processes, Frontiers in Zoology, Forest Ecology and Management y Molecular Ecology, entre otras. La guía PREPARE por su parte, entrega consideraciones para la planificación adecuada del estudio incluyendo el cumplimiento de las $3 R s$, realización de una valoración del daño-beneficio, justificación de cualquier posible efecto dañino sobre el animal, decisiones respecto a la unidad experimental y número, desarrollo de metodologías de captura, inmovilización, marcaje, liberación, translocación y administración de sustancias, entre otros manejos asociados al refinamiento (Smith et al. 2018). La adecuada implementación de las 3Rs deriva en el máximo bienestar animal, minimización de artefactos que podrían alterar los hallazgos, reducción de costos y una generación de conocimiento del más alto nivel.

\section{CONCLUSIÓN}

La atención a los detalles asociados a algunos aspectos del diseño experimental por parte de los CICUAs, como las 3Rs, busca tanto la promoción de la investigación de calidad y excelencia, como el resguardo ético del bienestar animal. No es su finalidad ser un trámite engorroso, ni menos afectar la viabilidad de las investigaciones como se plantea en D'Elia et al. (2019). De hecho, existe concordancia entre la comunidad científica y los CICUAs en varios aspectos del uso de animales, por ejemplo, en la remoción de individuos desde sus poblaciones naturales para su acabado estudio científico, actividad que debe considerar la implementación de las 3Rs. La ciencia y el desarrollo de conocimiento se beneficiarán en la medida que los investigadores y CICUAs se interioricen en los principios de las 3Rs y puedan conversar abiertamente, llevando a un proceso de evaluación y respaldo más expedito. Es por esto que invitamos a los(as) investigadores(as), académicos(as) y estudiantes, para que se acerquen a sus respectivos CICUAs y los vean como un organismo institucional que existe para apoyar y contribuir al desarrollo de una investigación científica de excelencia y alineada con los estándares éticos imperantes. Asimismo, hacemos un llamado a investigadores(as) cuyas líneas involucren FS a participar activamente de las labores de los CICUAs ya sea como miembros o a través de la colaboración directa en la revisión de protocolos que involucren manejo de especies silvestres.

Finalmente, sugerimos a los(as) investigadores(as) discutir responsablemente con sus colegas el valor científico y las consideraciones éticas de sus proyectos y líneas de investigación. Los CICUAs representan a la institución que aloja y patrocina a los proyectos y sus investigadores asociados, y por ende son parte de la investigación. Estos comités siempre estarán dispuestos a dialogar y mejorar los procedimientos que involucran el uso de animales, por el bien de la ciencia y su avance.

\section{AGRADECIMIENTOS}

Como representantes de diversos Comités de Bioética encargados del Cuidado y Uso de Animales, queremos reconocer y agradecer a todos los integrantes de dichos comités, que de manera voluntaria y desinteresada han aportado incansablemente al avance de la ciencia nacional, velando por el bienestar animal y la correcta conducción ética en las instituciones que realizan investigación.

\section{REFERENCIAS}

AWA. 1966. Animal Welfare Act (Laboratory Animal Welfare Act) of 1966. United States of America. P.L. 89-544.

ARRP. 2019. ARRP policies and guidelines. Animal Research Review Panel. Opportunistic research on free-living wildlife. Australia. URL: https://www.animalethics.org.au/ policies-and-guidelines/wildlife-research/opportunisticresearch

Carro, F., Pérez-Aranda, D., Lamosa, A., Schmalenberger, H.P., Pardavila, X., Gegúndez, M.I., Soriguer, R.C. 2007. Eficiencia de tres tipos de trampas para la captura de micromamíferos (Efficiency of three types of traps for trapping small mammals). Galemys 19: 73-81.

Chile. 1989. Ley General de Pesca y Acuicultura. Ministerio de Economía, Fomento y Reconstrucción, Chile.

Chile. 1996. Sustituye texto de la Ley N4601, Sobre Caza, y Artículo 609 del código civil. Ministerios de Agricultura, Chile.

Chile. 2009. Ley №20.380 Sobre Protección de Animales. Ministerio de Salud. Subsecretaría de Salud Pública, Chile.

Chile. 2016. Aplica Convención Sobre el Comercio Internacional de Especies Amenazadas de Flora y Fauna Silvestre. Ministerio de Agricultura, Chile.

Comisión Europea. 2012. Preocupándonos de los animales. Hacia una Ciencia mejor. Documento Marco para la Evaluación de la severidad. Directiva 2010/63/EU. 96 pp. URL: http://oir.umh.es/2016/03/08/documento-marcopara-la-evaluacion-de-la-severidad/

Crozier, G.K.D., Schulte-Hostedde, A.I. 2014. The ethical dimensions of wildlife disease management in an 
evolutionary context Evolutionary Applications 7(7): 788-798.

D’Elia, G., Jaksic, F., Bacigalupe, L.D., Bozinovic, F., Canto, J.L., Correa, C., Fontúrbel, F.E., Lisón, F., Méndez, M.A., Néspolo, R., Opazo, J.C., Palma, E., Rau, J.R., Rodríguez, S.M., Rodríguez-Serrano, E., Sabat, P., Vásquez, R.A., Victoriano, P. 2019. Sugerencias para mejorar la regulación chilena de manipulación de vertebrados terrestres en poblaciones naturales en el contexto de investigaciones científicas. Gayana 83(1): 63-67.

Directiva 2010/63/UE del Parlamento Europeo y del Consejo de 22 de septiembre de 2010 relativa a la protección de los animales utilizados para fines científicos. Diario Oficial de la Unión Europea. L 276/33.

Fraser, D., MacRae, A.M. 2011. Four types of activities that affect animals: implications for animal welfare science and animal ethics philosophy. Animal Welfare 20: 581-590.

Guidelines for the use of animals. 2012. Guidelines for the treatment of animals in behavioural research and teaching. Animal Behaviour 83: 301-309.

Honegger, R.E. 1979. Marking amphibians and reptiles for future identification. International Zoo Yearbook 19: 14-22.

Jessup, D.A. 2003. Opportunistic research and sampling combined with fish and wildlife management actions or crisis response. ILAR 44: 277-285.

Kilkenny, C., Browne, W.J., Cuthill, I.C., Emerson, M., Altman, D.G. 2010. Improving bioscience research reporting: The ARRIVE guidelines for reporting animal research. PLoS Biology 8(6): e1000412.

Lindsjö, J., Fahlman, Å., Törnqvist, E. 2016. Animal welfare from mouse to moose: implementing the principles of the 3rs in wildlife research. Journal of Wildlife Diseases $52(2$ Suppl): S65-77.

Loken, E., Gelman, A. 2017. Measurement error and the replication crisis. Science 355(6325): 584-585.

NRC. 2011. Guía para el cuidado y uso de animales de laboratorio.
Octava edición. Ediciones UC, Chile.

Porter, D.G. 1992. Ethical scores for animal experimentation. Nature 356: 101-102

Reed, B., Beatham, S., Carter, S., Clubb, R., Garrod, K., Gale, M., Gomm, M., Knight, K., Lane, J., Mathews, F., Pimlott, P., Smith, A., Wilson, R., Weyer, U., Hawkins, P. 2017. Report of a RSPCA/APHA meeting on the welfare of wild animals used in research. Animal Technology and Welfare 16(1): 13-25.

Redondo, T. 2012. Visión-Biológica. Aplicación del Principio 3Rs a la investigación con FS. Curso. Categoría B, EBD/CSIC y CEVUG/FEUGR. 15 p. URL: http://cevug.ugr.es/fauna_ silvestre

Russell, W.M.S., Burch, RL. 1959. The principles of humane experimental technique. Chapters 5, 6 7. Ed. Methuen, London, UK.

Sikes, R.S., Animal Care, Use Committee of the American Society of Mammalogists. 2016. Guidelines of the American Society of Mammalogists for the use of wild mammals in research and education. Journal of Mammalogy 97(3): 663-688.

Smith, A.J., Clutton, R.E., Lilley, E., Hansen, K.E.A., Brattelid, T. 2018. PREPARE: guidelines for planning animal research and testing. Laboratory Animals 52(2): 135-141.

Society for Conservation Biology. 2019. Code of Ethics. URL: https://conbio.org/about-scb/who-we-are/code-ofethics

Swart, J.A.A. 2004. The wild animals as a research animal. Journal of Agricultural and Environmental Ethics 17: 181-197.

Swart, J.A.A., Keulartz, J., 2011. Wild Animals in Our Backyard. A Contextual Approach to the Intrinsic Value of Animals. Acta Biotheoretica 59(2): 185-200.

Williams, R.M., Trites, A.W., Bain, D.E. 2002. Behavioural responses of killer whales (Orcinus orca) to whale-watching boats: opportunistic observations and experimental approaches. Journal of Zoology 256: 255-270.

Received: 30.05 .2019

Accepted: 11.01.2020 